\title{
Upper limb activity over time in complex regional pain syndrome type 1 as objectively measured with an upper limb-activity monitor: An explorative multiple case study
}

\author{
Fabiënne C. Schasfoort ${ }^{\text {a,* }}$, Johannes B.J. Bussmann ${ }^{\text {a }}$, H. John Krijnen ${ }^{\text {b }}$, Henk J. Stam ${ }^{\text {a }}$ \\ ${ }^{a}$ Department of Rehabilitation Medicine, Erasmus MC, University Medical Center Rotterdam, P.O. Box 1738, 3000DR Rotterdam, The Netherlands \\ ${ }^{\mathrm{b}}$ Department of Anesthesiology, Pain Clinic, Ikazia Hospital, Rotterdam, The Netherlands
}

Received 31 August 2004; accepted 17 January 2005

Available online 26 February 2005

\begin{abstract}
Background: An upper limb-activity monitor (ULAM) has been developed to determine activity limitations in complex regional pain syndrome type 1 (CRPS1). The ULAM is based on $24 \mathrm{~h}$ ambulatory monitoring of body segment accelerations and enables valid and objective quantification of mobility and upper limb activity in transversal studies.

Aims: To explore upper limb activity over time in acute upper limb CRPS1 as measured with the ULAM in a longitudinal study, and to compare this to time courses of other outcome measures for activity limitations and impairments.

Methods: Four subjects were measured four times during a treatment protocol. Several ULAM outcome measures related to upper limb usage and mobility, three questionnaires (RASQ, DASH, RAND36), and six impairment outcome indicators (VAS-momentary pain, VAS-pain resulting from effort, volume, temperature, active range of motion, strength) were used.

Results: Objectively measured upper limb activity frequently improved; improvements of $>5 \%$ were found for $63 \%$ of the ULAM outcome measures at final assessment. The ULAM outcome measures had a time course more similar to the body-part and CRPS1 specific questionnaire RASQ than the other questionnaires. The time course of impaired temperature was most often in accordance with the ULAM, and both VAS scores showed least accordance.

Conclusions: Clear changes in upper limb activity over time were frequently found as objectively measured with the ULAM, and relationships among the time courses of the ULAM and other outcome measures were largely explainable. The ULAM can validly assess upper limb activity over time in CRPS1, but between-measurement variability needs careful consideration.

(c) 2005 Published by Elsevier Ltd on behalf of European Federation of Chapters of the International Association for the study of Pain.
\end{abstract}

Keywords: Ambulatory accelerometry; Complex regional pain syndrome 1; Upper limb activity; Impairments; Activity limitations

\section{Introduction}

Complex regional pain syndrome type 1 (CRPS1) encompasses various impairments (Atkins et al., 1989; Schwartzman and Kerrigan, 1990) and usually leads to limitations of everyday activities and functioning. Until

\footnotetext{
* Corresponding author. Tel.: +31 104087588 ; fax: +31 104633843

E-mail address: f.schasfoort@erasmusmc.nl (F.C. Schasfoort).
}

recently, most CRPS1 research concentrated on impairments, and when activity limitations were quantified merely scales and questionnaires were used (Schasfoort et al., 2000). This lack of instruments to objectively measure activity limitations, together with the recently stressed importance of objective outcome measures for CRPS1 (Stanton-Hicks et al., 1998), and developments in ambulatory accelerometry (Bussmann et al., 2001) formed the basis of the upper limb-activity monitor Pain. 
(ULAM). The device is based on long-term ambulatory monitoring of signals from body-fixed acceleration sensors and allows detailed objective quantification of mobility-related activities and upper limb activity during everyday life (Schasfoort et al., 2002). The ULAM is an extension of a validated activity monitor (AM) (Tulen et al., 1997; Bussmann et al., 1998a,b,c; van den BergEmons et al., 2000) and consists of sensors on forearms, thighs and trunk, connected to a waist-worn recorder (Fig. 1).

In chronic upper limb CRPS1 patients, the ULAM detected limited intensity, percentage and proportion of upper limb activity when compared to healthy subjects (Schasfoort et al., 2003), impaired grip strength and active range of motion of wrist and digits explained most variability in activity limitations as measured with the ULAM (Schasfoort et al., 2004), and a study of relationships between ULAM and questionnaires demonstrated that the device offers an alternative but important insight into everyday functioning (Schasfoort et al., 2005). In short, the ULAM has proven feasibility, validity and relevance in transversal group studies in chronic upper limb CRPS1.

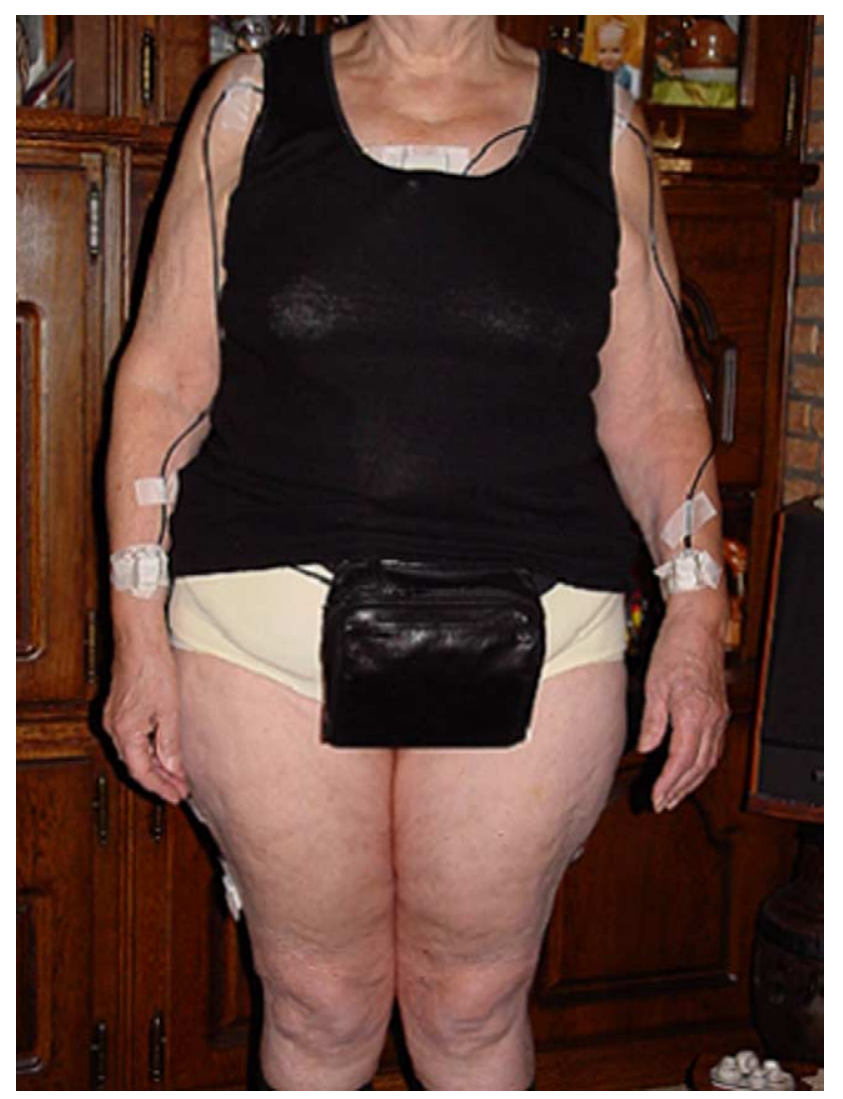

Fig. 1. A subject wearing the ULAM in her home environment. Several body-fixed piezo-resistive acceleration sensors (ADXL202, size $1 \times 1 \times 0.5 \mathrm{~cm}$, Analog Devices, Breda, the Netherlands) are connected to a waist-worn recorder (Temec Instruments BV, Kerkrade, The Netherlands).
Usage of the ULAM has not yet been explored longitudinally on either the individual patient or the group level, however. It is unknown whether the ULAM can detect changes in upper limb activity over time, or how changes as measured with the ULAM are related to changes over time as measured with other instruments (assuming that if functioning changes over time, this is reflected in both ULAM and other instruments). The ULAMs feasibility to detect changes is important for CRPS1 research and practice because it enables objective monitoring of treatment effects on everyday functioning in future (intervention) studies. Therefore, the research questions were:

- What is the time course of upper limb activity as objectively measured with the ULAM in acute upper limb CRPS1?

- Is the time course of upper limb activity as measured with the ULAM related to the time courses of other instruments and outcome measures at the activity and impairment levels?

- Which practical and methodological issues have to be dealt with before the ULAM can be validly used in future longitudinal (intervention) studies?

\section{Materials and methods}

\subsection{Design, subjects and treatment regimen}

Because changes are more likely in acute than in chronic CRPS1, subjects having upper limb CRPS1 for less than 10 weeks were recruited from the pain clinic (H.J.K.) for this explorative study. CRPS1 was diagnosed according to Veldman's criteria (Veldman et al., 1993) that are similar to those of the IASP (StantonHicks et al., 1995; Bruehl et al., 1999).

Four subjects with fractures as causative event volunteered. Subject A was a 48-year old man with non-dominant side CRPS1 who worked with the police detective force, was divorced and lived with his two children. Subject $\mathrm{B}$ was a 58 -year old man with non-dominant side CRPS1, worked as a mechanic, was married and lived with his wife and child. Subject C was a 71-year old woman with non-dominant side CRPS1, was married and lived with husband. Subject D was a 52-year old man with dominant side CRPS1, worked as a night watchman, was married and lived with his wife and two children.

Since there still is no well-accepted evidence based treatment algorithm for CRPS1 (Stanton-Hicks et al., 1998; Goris, 2001; Ribbers and Stam, 2001), and treatment efficacy to reduce impairments and/or activity limitations has scarcely been demonstrated with methodologically sound studies (Perez et al., 2001; Oerlemans 
et al., 2000b; Schasfoort et al., 2000), we used a reasonably standardized treatment regimen that aimed at improving impairments and everyday functioning. Treatment parameters included radical scavenger vitamin C, capillary vasodilator ketanserin, large vessel vasodilator verapamil, aerobe metabolism stimulator and radical scavenger carnitine, guanethinide iontoforesis, carnitine infusion, physical therapy exercises and connective tissue massage, and manual therapy with a segmental approach aimed at improving functioning.

The subjects were assessed four times at roughly the same points during their treatment, with at least two weeks between measurements; $t_{0}$ within two days after first visit to the pain clinic, $t_{1}$ just before a series of iontoforesis and infusion, $t_{2}$ just after a series of iontoforesis and infusion, and $t_{3}$ at least four weeks after $t_{2}$. Due to hospital logistics, however, subject D had two series of iontoforesis and infusion between $t_{0}-t_{1}$ and $\mathrm{t}_{2}-\mathrm{t}_{3}$.

\subsection{Instruments and outcome measures}

The ULAM objectively determined whether or not the upper limbs are active when a subject is performing one of these mobility-related activities: lying, sitting, standing, walking, cycling and general movement (Schasfoort et al., 2002). The measurement technique and automated signal analysis with 'feature' signals derived from raw acceleration signals have been described extensively (Bussmann et al., 1998a,b,c; van den BergEmons et al., 2000, 2001a,b; Bussmann et al., 2001; Schasfoort et al., 2002). The following ULAM outcome measures, with lower scores indicating more limited activity, were used (Schasfoort et al., 2003; Schasfoort et al., 2004):

- \%dyn: the percentage of the measurement period that dynamic mobility-related activities (i.e., the body motions walking, cycling and general non-cyclic activity) were performed;

- intsit and intstand: the mean intensity of upper limb activity of the involved side while subjects were sitting and standing, expressed in scaled $\mathrm{g}\left(9.81 \mathrm{~ms}^{-2}\right)$;

- \%sit and \%stand: the percentage of the time that the involved upper limb was active while subjects were sitting and standing.

Because there was no 'gold standard' for objective measurement of activity limitations over time and changes were not guaranteed, the time courses of the ULAM outcome measures were compared to the courses of questionnaire outcome measures. We used three questionnaires that also aim at limitations of everyday functioning, but with other measuring techniques and different operationalizations of the concept functioning than the ULAM (Schasfoort et al., 2005). It was assumed that if functioning changed over time, this had to be reflected to a lesser or greater extent in both ULAM and questionnaires, depending on the strength of the conceptual relationships between the instruments and similarities regarding instrument characteristics. The following questionnaires were used:

- RAND36 health survey: the RAND36 (VanderZee et al., 1996) is a generic questionnaire that has been used in CRPS1 research (Geertzen et al., 1998a,b). Although, it was most responsive of five generic questionnaires (Beaton et al., 1997), it was less responsive for upper limb disorders (Amadio et al., 1996) because of ceiling- and floor-effects;

- Radboud skills questionnaire: the Radboud skills questionnaire (RASQ) is a reliable body-part specific questionnaire (Oerlemans et al., 2000a) especially developed for upper limb CRPS1 that compares the current effort certain activities cost to pre-CRPS1 (Oerlemans et al., 2000b). Its responsiveness has not been studied specifically;

- Disabilities of arm shoulder and hand questionnaire: the disabilities of arm shoulder and hand questionnaire (DASH) function and symptom score is a 30-item body-part specific questionnaire that mainly measures limitations of everyday activity in upper limb disorders (Hudak et al., 1996; Davis et al., 1999; Veehof et al., 2002) but also contains some impairment items. Responsiveness was sufficient after carpal tunnel release (Gay et al., 2003).

Time courses of six impairment outcome indicators, that have been described as a responsive multi-component score for (acute) upper limb CRPS1 (Oerlemans et al., 1998; Oerlemans et al., 2000b), were also explored:

- Visual analogue scale-effort: pain intensity resulting from effort measured with a visual analogue scale (VAS);

- VAS-moment: momentary pain intensity measured with a VAS;

- Volume: the difference in volumetric measurements (edema, atrophy) between both hands in relation to the volume of the non-involved side measured with a fluid overflow volumeter (Volumeters Unlimited, Idywild, USA);

- Temperature: temperature of the dorsal side of the involved hand relative to the non-involved side measured with an infrared thermometer (Braun Pro 3000 Type 6014, Kronberg, Germany);

- Active range of motion: maximum active range of motion (AROM) within pain threshold of the wrist and two most impaired fingers of the involved hand relative to the non-involved side measured with a goniometer; 
- Strength: four point grip strength of the involved hand relative to the non-involved side measured with a portable hand-held dynamometer (Microfet, Hoggan Health Industries Inc., Draper, USA).

Impairment outcome indicator scores were converted to a range of $1-10$ with a score of 1 to be interpreted as absence of that impairment and 10 as severely impaired. For more information we refer to other studies (Oerlemans et al., 1998; Schasfoort et al., 2004).

\subsection{Protocol}

Assessments were in the subjects' home environment. Informed consent was signed, the ULAM was fitted and information regarding treatment obtained. The subjects were instructed to continue everyday activities while wearing the ULAM for $24 \mathrm{~h}$, except for swimming, bathing or showering. After removal, the questionnaire and impairment scores were obtained and subjects were asked about ULAM wearing comfort and possible influences of wearing it on their behavior. Furthermore, we asked whether the wearing period was representative for other weekdays, and a gross overview of activities performed was noted to support data interpretation. The exact ULAM measurement technique and output parameters were not explained until final assessment. All subjects agreed with this protocol, which was approved by the local medical ethical committees.

\subsection{Data analysis}

The absolute values of the ULAM outcome measures were analyzed at assessments $t_{0}-t_{3}$ to determine the time course of objectively measured upper limb activity. Subsequently, changes in the absolute values of all outcome measures at $t_{1}, t_{2}$ and $t_{3}$ compared to baseline $\left(t_{0}\right)$ were normalized to visualize courses in time: the maximum absolute change compared to baseline (either for time interval $t_{1}-t_{0}, t_{2}-t_{0}$ or $t_{3}-t_{0}$, either positive or negative, either for subject A, B, C or D) was set at $+100 \%$ (or $-100 \%$ in case of a negative change).

These normalized change scores were shown in bar graphs for both the individual subjects and the group $(n=4)$. A from a clinical viewpoint 'ideal pattern' for time courses of an outcome measure would be that the normalized time interval for $t_{3}-t_{0}$ showed the highest percentage, whereas the $t_{2}-t_{0}$ and $t_{1}-t_{0}$ intervals both showed, respectively, lower percentages. Such a pattern means that the more time had passed, the more a subject's functioning had improved (irrespective of whether positive changes were due to treatment or natural recovery).

These bar graphs displays differences between subjects with respect to both magnitude and direction of changes over time for each of the outcome measures (because only one interval for one subject was set at $100 \%$ ). It has to be noticed, however, that these bar graphs only display differences between outcome measures with respect to direction of changes over time and not magnitude (i.e., due to the normalization method, the absolute value of the $100 \%$ normalized change score of one outcome measure could theoretically be smaller than the absolute value of a $10 \%$ normalized change score of another outcome measure).

To compare time courses we calculated how often changes over time between consecutive follow-up assessments $\left(t_{1}-t_{0}, t_{2}-t_{1}\right.$ and $\left.t_{3}-t_{2}\right)$ as measured with the ULAM were in the same direction as changes over time as measured with the other outcome measures. For each combination of two outcome measures twelve delta pairs ( 4 assessments, so 3 deltas for each of the 4 subjects) were analyzed. The higher the number of changes in the same direction for two outcome measures (either positive or negative), the more similar the time courses for these outcome measures. This simple approach for data analysis was in our opinion appropriate considering the relatively new ULAM measurement technique, the low number of subjects, the explorative character of the study, and the research questions.

\section{Results}

The subjects did not report any problems wearing the ULAM although they had to get used to it for a few minutes each time it was fitted. Five of the 12 measurement days were not representative according to the patient's own opinion (Table 1). This was due to unusual overactivity such as organizing a barbeque party or unusual inactivity such as going to a lecture, taking an unexpected day off from work, or hot humid weather.

\subsection{Time course of objectively measured upper limb activity}

For subject A, all ULAM outcome measures demonstrated improvements at the end of the 3-months measurement period compared to baseline; intsit $+24 \%$, $\%$ sit $+28 \%$, intstand $+25 \%$, $\%$ stand $+7 \%$ (Table 1 ). At final assessment, subject $\mathrm{A}$ was working full-time and started jogging again. Subject B's upper limb activity as measured with the ULAM had hardly changed at the end of his 3-month measurement period compared to baseline (intsit $+7 \%$, \%sit $-2 \%$, intstand $+4 \%$, $\%$ stand $+1 \%$ ). It has to be noted that his treatment was complicated with a delayed start and premature ending of physical therapy in addition to an unintentional too small initial dose of medication. For subject $\mathrm{C}$, each ULAM upper limb outcome measure indicated improved functioning between $t_{0}$ and $t_{1}$, and was relatively stable at $t_{2}$ and $t_{3}$. At the end of the 4-month 
Table 1

Overview of absolute values for the ULAM outcome measures, the questionnaire outcome measures and the impairment outcome indicators of the four individual subjects

\begin{tabular}{|c|c|c|c|c|c|c|c|c|c|c|c|c|c|c|c|c|}
\hline & \multicolumn{4}{|c|}{ Subject A } & \multicolumn{4}{|c|}{ Subject B } & \multicolumn{4}{|c|}{ Subject $\mathrm{C}$} & \multicolumn{4}{|c|}{ Subject D } \\
\hline & $\mathrm{t}_{0}$ & $\mathrm{t}_{1}$ & $\mathrm{t}_{2}^{-}$ & $\mathrm{t}_{3}^{-}$ & $\mathrm{t}_{0}$ & $t_{1}$ & $t_{2}$ & $t_{3}$ & $\mathrm{t}_{0}$ & $\mathrm{t}_{1}^{-}$ & $t_{2}$ & $t_{3}$ & $\mathrm{t}_{0}^{+}$ & $t_{1}$ & $t_{2}$ & $\mathrm{t}_{3}^{-}$ \\
\hline \multicolumn{17}{|c|}{ ULAM activity outcome measures } \\
\hline \multicolumn{17}{|c|}{ The higher the score, the better the functioning } \\
\hline$\%$ dyn & 9,8 & 10,1 & $11,8^{\circ}$ & 10,6 & 11,8 & 16,6 & 10,3 & 11,8 & 7,0 & 6,8 & 7,0 & 9,4 & 19,5 & 12,3 & 17,4 & 13,8 \\
\hline intsit & 2,8 & 4,0 & 4,1 & 3,5 & 3,4 & 2,8 & 3,7 & 3,6 & 1,9 & 5,1 & 5,2 & 4,9 & 2,4 & 1,9 & 2,6 & 2,4 \\
\hline$\%$ sit & 27,6 & 40,4 & 35,3 & 35,2 & 37,1 & 31,0 & 39,1 & 36,4 & 20,3 & 51,4 & 45,4 & 45,1 & 23,0 & 19,5 & 26,6 & 22,2 \\
\hline intstand & 6,3 & 7,1 & 7,6 & 7,9 & 8,0 & 8,3 & 9,7 & 8,3 & 9,1 & 10,7 & 11,0 & 11,3 & 6,8 & 8,4 & 8,7 & 9,0 \\
\hline$\%$ stand & 53,7 & 56,1 & 55,9 & 57,4 & 73,8 & 73,6 & 78,5 & 74,7 & 79,0 & 82,4 & 77,9 & 80,2 & 68,3 & 81,9 & 76,6 & 74,4 \\
\hline \multicolumn{17}{|c|}{$\begin{array}{l}\text { Questionnaire activity outcome measures } \\
\text { The lower the score, the better the functioning }\end{array}$} \\
\hline RASQ (1-5) & 2,5 & 1,8 & 1,3 & 1,2 & 2,8 & 2,7 & 2,3 & 2,4 & 3,8 & 2,7 & 2,3 & 2,0 & 2,9 & 2,4 & 2,1 & 1,6 \\
\hline DASH $(0-100)$ & 41 & 44 & 24 & 15 & 42 & 39 & 50 & 41 & 67 & 56 & 50 & 50 & 57 & 50 & 41 & 34 \\
\hline \multicolumn{17}{|c|}{ The higher the score, the better the functioning } \\
\hline RAND36 (0-100) & 62 & 68 & 71 & 79 & 61 & 51 & 59 & 61 & 33 & 40 & 56 & 49 & 69 & 65 & 62 & 70 \\
\hline \multicolumn{17}{|c|}{$\begin{array}{l}\text { Impairment indicator outcome measures } \\
\text { The lower the score, the better the functioning }\end{array}$} \\
\hline VAS effort $(1-10)$ & 2 & 3 & 1 & 1 & 2 & 5 & 5 & 4 & 4 & 4 & 4 & 3 & 7 & 3 & 4 & 2 \\
\hline VAS moment (1-10) & 1 & 1 & 1 & 1 & 1 & 2 & 5 & 4 & 3 & 2 & 3 & 3 & 2 & 2 & 2 & 1 \\
\hline Volume $(1-10)$ & 5 & 4 & 4 & 4 & 1 & 1 & 1 & 1 & 6 & 4 & 1 & 2 & 5 & 3 & 1 & 1 \\
\hline Temperature (1-10) & 2 & 5 & 3 & 4 & 2 & 3 & 5 & 3 & 4 & 2 & 1 & 3 & 4 & 1 & 1 & 2 \\
\hline AROM (1-10) & 7 & 7 & 6 & 5 & 3 & 2 & 2 & 2 & 8 & 6 & 6 & 5 & 6 & 3 & 2 & 2 \\
\hline Strength $(1-10)$ & 6 & 4 & 3 & 3 & 6 & 4 & 4 & 5 & 7 & 7 & 5 & 5 & 6 & 4 & 5 & 4 \\
\hline
\end{tabular}

$\mathrm{T}_{n}^{-}$unrepresentative 'inactive' ULAM $24 \mathrm{~h}, \mathrm{~T}_{n}^{+}$unrepresentative 'overactive' $24 \mathrm{~h}$ ULAM.

period, all ULAM outcome measures indicated improved functioning; intsit $+158 \%$, \% sit $+123 \%$, intstand $+25 \%$, \%stand $+2 \%$. Subject D's upper limb activity during sitting had hardly changed compared to baseline (intsit $+1 \%$, \%sit $-4 \%$ ), whereas the ULAM outcome measures during standing indicated improved functioning despite unfavorable unrepresentativeness (intstand $+32 \%$, \%stand $+9 \%$ ). Improvements of $>5 \%$ between baseline and final assessments were found for the majority of ULAM upper limb outcome measures (10/16, $63 \%$ ) in these four subjects with acute upper limb CRPS1.

\subsection{Time courses of normalized change scores}

The normalized change score patterns were "clinically ideal' only for few outcome measures (Fig. 2, see also Section 2); actually primarily for the ULAM outcome measure intstand, the RASQ, and the impairment outcome indicator AROM. Questionnaires more often displayed a clinically ideal pattern than the ULAM that, in turn, more often displayed such a pattern than the impairment outcome indicators. Not one "clinically ideal' pattern was found for subject $\mathrm{B}$; his normalized change scores were divergent from the other subjects. For subjects A, C and D, the majority of questionnaire and impairment outcome measures displayed their maximum normalized positive change for the $t_{3}-t_{0}$ time interval, which was not true for the ULAM outcome measures. Outcome measures that displayed a negative normalized change or did not or hardly change during two or more of the time intervals were mainly at the impairment level, and mainly for subject B. The ULAM outcome measure \%dyn appeared dissimilar to the other ULAM outcome measures.

\subsection{Time courses of the ULAM outcome measures in relation to other outcome measures}

Calculations of how often changes over time between consecutive assessments $\left(t_{1}-t_{0}, t_{2}-t_{1}\right.$ and $\left.t_{3}-t_{2}\right)$ were in the same direction (Table 2) showed that the time courses of ULAM outcome measures were most often in the same direction with the RASQ. The time course of the ULAM outcome measures intstand was overall best related to the time courses of both the questionnaire and impairment outcome measures (with a maximum score of 12 changes in the same direction with the RASQ).

\section{Discussion}

Despite actually unchanged functioning in subject B and the sometimes very short (i.e., two weeks) time intervals between assessments, objectively measured improvements of $>5 \%$ between baseline and final assessments were found for the majority of ULAM upper limb 

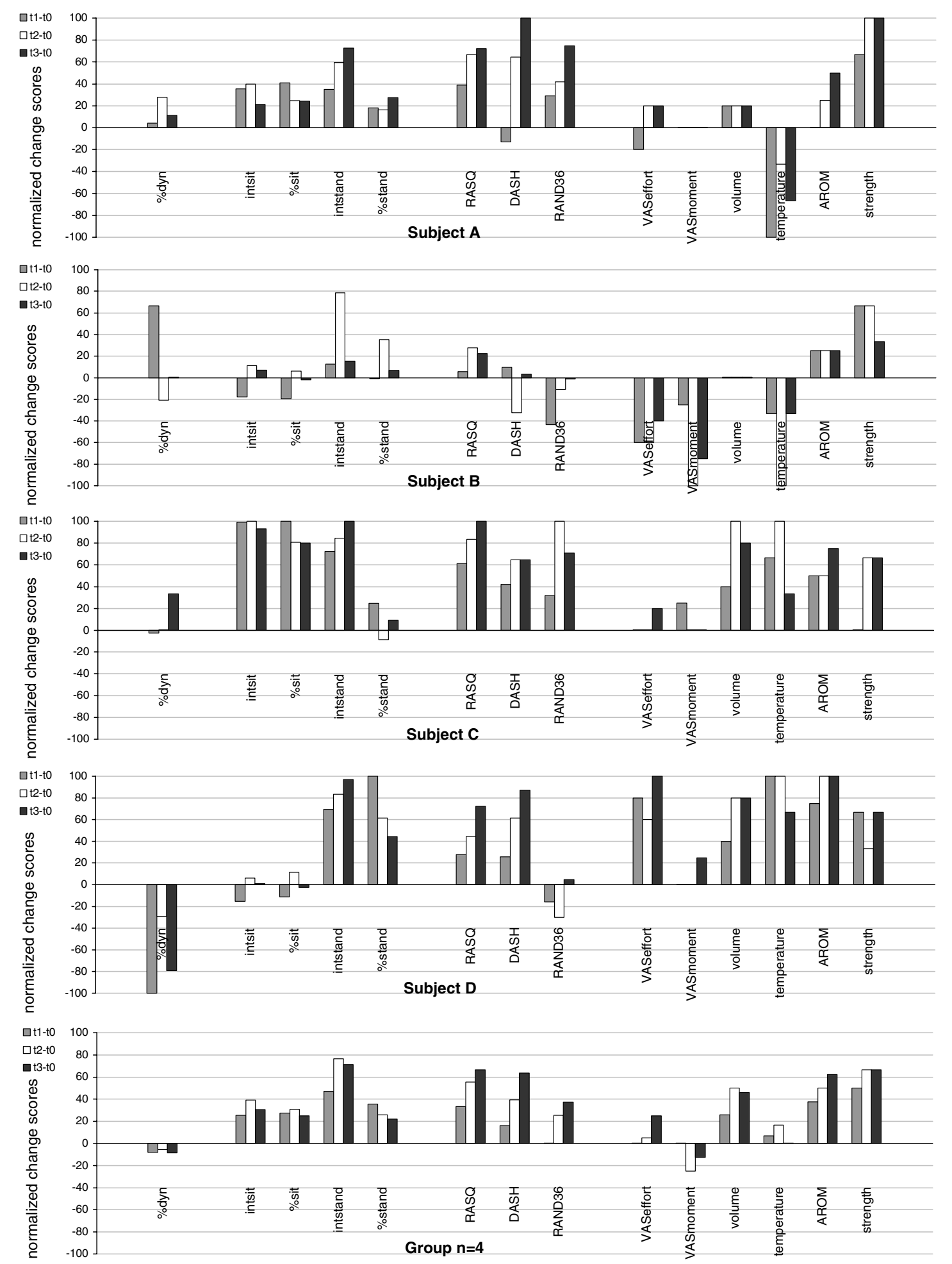

Fig. 2. Overview of normalized change scores of all outcome measures for time intervals $t_{1}-t_{0}, t_{2}-t_{0}$ and $t_{3}-t_{0}$, for the individual subjects and the average score of these subjects as a small group. The maximum absolute change compared to baseline (either for time interval $t_{1}-t_{0}, t_{2}-t_{0}$ or $t_{3}-t_{0}$, either positive or negative, either for subject A, B, C or D) was set at $+100 \%$ (or $-100 \%$ in case of a negative change).

outcome measures. Because we had no idea as to how limited the subjects would be or how fast changes over time would occur, the small time frames were considered appropriate for the current longitudinal study, however. The divergent time course of \%dyn was anticipated con- sidering the population studied; the subjects had an upper limb disorder and were not limited with respect to mobility. This was also found in chronic upper limb CRPS1 (Schasfoort et al., 2003). The present 11-12 \%dyn did not differ from healthy subjects (Bussmann 
Table 2

The time courses of the five ULAM outcome measures in relation to the time courses of the questionnaire and impairment outcome measures

\begin{tabular}{lccccccccccc}
\hline & RAND 36 & RASQ & DASH & Sum & VAS effort & VAS moment & Volume & Temperature & AROM & Strength & Sum \\
\hline \%dyn & 5 & 6 & 6 & 17 & 3 & 2 & 3 & 6 & 4 & 4 & 22 \\
intsit & 8 & 7 & 4 & 19 & 2 & 2 & 5 & 7 & 3 & 4 & 23 \\
\%sit & 6 & 5 & 2 & 13 & 1 & 3 & 4 & 5 & 2 & 2 & 17 \\
intstand & 7 & 12 & 8 & 27 & 4 & 2 & 5 & 4 & 7 & 7 & 29 \\
\%stand & 6 & 7 & 3 & 16 & 4 & 3 & 3 & 4 & 4 & 22 \\
Sum & 32 & 37 & 23 & & 14 & 12 & 20 & 26 & 20 & 21 \\
\hline
\end{tabular}

It was calculated how often changes over time between asessments $\left(t_{1}-t_{0}, t_{2}-t_{1}\right.$ and $\left.t_{3}-t_{2}\right)$ as measured with the ULAM outcome measure were in the same direction as changes over time as measured with the other outcome measures (maximum per pair of outcome measures is 12).

et al., 1998a,b,c; van den Berg-Emons et al., 2000, 2001a,b; Bussmann et al., 2001; Schasfoort et al., 2003). Although, these four subjects showed more limited upper limb activity than 30 subjects with chronic CRPS1 previously measured with the ULAM (Schasfoort et al., 2003, 2004, 2005), this should not be interpreted as a confirmation of the supposition that functioning is generally more limited in acute than in chronic CRPS1. CRPS1 is a syndrome and varies enormously between subjects with respect to type and magnitude of impairments and activity limitations, as well as the duration of these consequences.

The finding that the body-part specific RASQ had a time course more similar to the ULAM than the RAND36 and DASH was probably due to more similarities with respect to operationalization of functioning and other instrument characteristics, as also found in our transversal studies in chronic CRPS1 (Schasfoort et al., 2005). Changes over time measured with impairment outcome indicators were less well related to changes as measured with the ULAM than the questionnaires were. This was not surprising because impairments are operationalizations of functioning at a different level than ULAM and questionnaires (i.e., body impairments versus a person's activity). Among the impairments, the time courses of volume, temperature, AROM and strength were more frequently in concert with the ULAM than the VAS pain scores, which may be explained by widely varying pain levels during the day in acute CRPS1 (Oerlemans et al., 1998). It has to be noticed that the present VAS scores were not high; the subjects may not have been representative for acute CRPS1 with respect to pain intensity.

With respect to the third research question it appeared that between-measurement variability is an important methodological issue of ULAM measurements. Variability between measurements can be resulting from several factors. First, everyone's level of everyday physical activity will vary, even within 'regular days', and thus the level of upper limb activity in CRPS patients will also vary. The intra-individual biological variability of upper limb activity as measured with the ULAM could not yet be investigated because we have only performed 24-h measurements. Between-day variability for \%dyn has been studied in 48-h measurements with the ULAMs older sibling AM (van den BergEmons et al., 2001a,b), however, and appeared to be $1.1 \%$ and $1.3 \%$ in two different patient groups and $0.8 \%$ in healthy subjects. Some between-day variability for ULAM data will not be problematic as long as it is relatively small compared to actual changes. Intraand inter-individual between-day variability have to be studied for both patients and healthy subjects to determine to which degree changed upper limb activity as measured with the ULAM falls under biological variability and above which threshold changed upper limb activity can be considered as clinically relevant. This issue is important to determine the optimal number of monitoring days and the required sample size for future studies, and is also related to anticipated treatment efficacy and the time period between assessments in future longitudinal studies. Of course, the current data do not allow us to make statements on treatment efficacy.

Second, besides random fluctuation within a probably limited range, some days may clearly be different from 'regular' days. This unrepresentativeness due to unusual overactivity or inactivity was reflected in the ULAM data. Because questionnaires measure a perceived average score over the last few days whereas the ULAM measured what subjects actually did during a specific $24-\mathrm{h}$ period, such unrepresentativeness was logically not (or less) reflected in the questionnaire scores. Concerning possible negative effects of unrepresentativeness of the measurement day on validity, it will stay important to ask a patient about this matter. Possibly, measurement days that are not representative should not be included in the analysis in future studies. Increasing the number of measurement days will also address this issue.

Third, the instrument itself can be a source of withinmeasurement variability. The ULAM upper limb outcome measures are rather rough; it detects upper limb activity but as a consequence of the measurement technique does not yet allow valid measurement of every aspect of upper limb usage (Schasfoort et al., 2002). Due to our experiences and data from previous studies, however, we think that this latter point is less important than 
the issue of between-day variability in everyday physical activity.

In conclusion, clear changes in upper limb activity over time were frequently found as objectively measured with the ULAM. The relationships between the time courses of the ULAM outcome measures and the time courses of other outcome measures for activity limitations and impairments appeared logical and for a great deal explainable. These results, in our opinion, demonstrate that the current ULAM has potential to objectively and validly assess changes in upper limb activity over time in future longitudinal (treatment efficacy) studies in upper limb CRPS1. It has to be realized, however, that between-measurement variability in upper limb activity is a factor that needs careful consideration during data analysis, and especially during analyses at the individual patient level.

\section{References}

Amadio PC, Silverstein MD, Ilstrup DM, Schleck CD, Jensen LM. Outcome assessment for carpal tunnel surgery: the relative responsiveness of generic, arthritis-specific, disease-specific, and physical examination measures. J Hand Surg [Am] 1996;21: 338-46.

Atkins RM, Duckworth T, Kanis JA. Algodystrophy following Colles' fracture. J Hand Surg [Br] 1989;14:161-4.

Beaton DE, Hogg-Johnson S, Bombardier C. Evaluating changes in health status: reliability and responsiveness of five generic health status measures in workers with musculoskeletal disorders. J Clin Epidemiol 1997;50:79-93.

Bruehl S, Harden RN, Galer BS, Saltz S, Bertram M, Backonja M, et al. External validation of IASP diagnostic criteria for complex regional pain syndrome and proposed research diagnostic criteria. International association for the study of pain. Pain 1999;81:147-54.

Bussmann HB, Reuvekamp PJ, Veltink PH, Martens WL, Stam HJ. Validity and reliability of measurements obtained with an activity monitor in people with and without a transtibial amputation. Phys Ther 1998;78:989-98.

Bussmann JB, Tulen JH, van Herel EC, Stam HJ. Quantification of physical activities by means of ambulatory accelerometry: a validation study. Psychophysiology 1998;35:488-96.

Bussmann JB, van de Laar YM, Neeleman MP, Stam HJ. Ambulatory accelerometry to quantify motor behaviour in patients after failed back surgery: a validation study. Pain 1998;74:153-61.

Bussmann JBJ, Martens WLJ, Tulen JHM, Schasfoort FC, BergEmons HJGvd, Stam HJ. Measuring daily behaviour using ambulatory accelerometry: the activity monitor. Behav Res Method Instrum Comp 2001;33:349-56.

Davis AM, Beaton DE, Hudak P, Amadio P, Bombardier C, Cole D, et al. Measuring disability of the upper extremity: a rationale supporting the use of a regional outcome measure. $\mathrm{J}$ Hand Ther 1999;12:269-74.

Gay RE, Amadio PC, Johnson JC. Comparative responsiveness of the disabilities of the arm, shoulder, and hand, the carpal tunnel questionnaire, and the SF-36 to clinical change after carpal tunnel release. J Hand Surg [Am] 2003;28:250-4.

Geertzen JH, Dijkstra PU, Groothoff JW, ten Duis HJ, Eisma WH. Reflex sympathetic dystrophy of the upper extremity - a 5.5-year follow- up. Part II. Social life events, general health and changes in occupation. Acta Orthop Scand 1998;279(Suppl.):19-23.
Geertzen JH, Dijkstra PU, van Sonderen EL, Groothoff JW, ten Duis HJ, Eisma WH. Relationship between impairments, disability and handicap in reflex sympathetic dystrophy patients: a long-term follow-up study [In Process Citation]. Clin Rehabil 1998;12:402-12.

Goris RJA. Behandelingsprotocol acute posttraumatische dystrofie, Treatment protocol acute complex regional pain syndrome type I, Nijmegen, 2001

Hudak PL, Amadio PC, Bombardier C. Development of an upper extremity outcome measure: the DASH (disabilities of the arm, shoulder and hand) [corrected]. The upper extremity collaborative group (UECG). Am J Ind Med 1996;29:602-8.

Oerlemans HM, Cup EH, DeBoo T, Goris RJ, Oostendorp RA. The Radboud skills questionnaire: construction and reliability in patients with reflex sympathetic dystrophy of one upper extremity. Disabil Rehabil 2000;22:233-45.

Oerlemans HM, Goris RJ, Oostendorp RA. Impairment level sumscore in reflex sympathetic dystrophy of one upper extremity. Arch Phys Med Rehabil 1998;79:979-90.

Oerlemans HM, Oostendorp RA, de Boo T, van der Laan L, Severens JL, Goris JA. Adjuvant physical therapy versus occupational therapy in patients with reflex sympathetic dystrophy/complex regional pain syndrome type I. Arch Phys Med Rehabil 2000;81:49-56.

Perez RS, Kwakkel G, Zuurmond WW, de Lange JJ. Treatment of reflex sympathetic dystrophy (CRPS type 1): a research synthesis of 21 randomized clinical trials. J Pain Symptom Manage 2001;21:511-26.

Ribbers GM, Stam HJ. Complex regional pain syndrome type I treated with topical capsaicin: a case report. Arch Phys Med Rehabil 2001;82:851-2.

Schasfoort FC, Bussmann JB, Stam HJ. Outcome measures for complex regional pain syndrome type I: an overview in the context of the international classification of impairments, disabilities and handicaps. Disabil Rehabil 2000;22:387-98.

Schasfoort FC, Bussmann JB, Stam HJ. Ambulatory measurement of upper limb usage and mobility-related activities during normal daily life with an upper limb-activity monitor: a feasibility study. Med Biol Eng Comput 2002;40:173-82.

Schasfoort FC, Bussmann JBJ, Stam HJ. Impairments and activity limitations in patients with upper limb complex regional pain syndrome type I. Arch Phys Med Rehabil 2004;85:557-66.

Schasfoort FC, Bussmann JBJ, Stam HJ. Relationships between a novel upper limb-activity monitor and four other instruments to determine functioning in upper limb complex regional pain syndrome type I. J Rehabil Med, 2005 [in press].

Schasfoort FC, Bussmann JBJ, Zandbergen AMAJ, Stam HJ. Impact of upper limb complex regional pain syndrome type I on everyday life measured with a novel upper limb-activity monitor. Pain 2003;101:79-88.

Schwartzman RJ, Kerrigan J. The movement disorder of reflex sympathetic dystrophy. Neurology 1990;40:57-61.

Stanton-Hicks M, Baron R, Boas R, Gordh T, Harden N, Hendler N, et al. Complex regional pain syndromes: guidelines for therapy. Clin J Pain 1998;14:155-66.

Stanton-Hicks M, Janig W, Hassenbusch S, Haddox JD, Boas R, Wilson P. Reflex sympathetic dystrophy: changing concepts and taxonomy. Pain 1995;63:127-33.

Tulen JH, Bussmann HB, van Steenis HG, Pepplinkhuizen L, Man in 't Veld AJ. A novel tool to quantify physical activities: ambulatory accelerometry in psychopharmacology. J Clin Psychopharmacol 1997;17:202-7.

van den Berg-Emons HJG, Bussmann JBJ, Balk AHMM, Stam HJ. Validity of ambulatory accelerometry to quantify physical activity in heart failure. Scand J Rehab Med 2000;32:187-92.

van den Berg-Emons HJG, Bussmann JBJ, Balk A, Keijzer-Oster D, Stam HJ. Level of activities associated with mobility during 
everyday life in patients with chronic congestive heart failure as measured with an activity monitor. Phys Ther 2001;81:1502-11.

van den Berg-Emons HJG, Bussmann JBJ, Brobbel AS, Roebroeck ME, Meeteren Jv, Stam HJ. Everyday physical activity in adolescents and young adults with meningomyelocele as measured with a novel activity monitor. J Pediatr 2001;139:880-6.

VanderZee KI, Sanderman R, Heyink J. A comparison of two multidimensional measures of health status: the Nottingham health profile and the RAND 36-item health survey 1.0. Qual Life Res 1996;5:165-74.

Veehof MM, Sleegers EJ, van Veldhoven NH, Schuurman AH, van Meeteren NL. Psychometric qualities of the Dutch language version of the disabilities of the arm, shoulder, and hand questionnaire (DASH-DLV). J Hand Ther 2002;15:347-54.

Veldman PH, Reynen HM, Arntz IE, Goris RJ. Signs and symptoms of reflex sympathetic dystrophy: prospective study of 829 patients. Lancet 1993;342:1012-6. 